\title{
Promoting Interspecies Electron Transfer with Biochar
}

SUBJECT AREAS:

ENVIRONMENTAL

MICROBIOLOGY

APPLIED MICROBIOLOGY

Received

19 November 2013

Accepted

25 April 2014

Published

21 May 2014

Correspondence and requests for materials should be addressed to

S.C. 1447681880@

qq.com) or A.-E.R.

larotaru@microbio.

umass.edu; arotaru@

biology.sdu.dk)

* Current address:

Nordic Center for Earth

Evolution, University of

Southern Denmark,

Campussvej 55,

Odense M, DK-5230,

Denmark.

† Current address:

Energy Biosciences

Institute University of

California Berkeley,

CA 94704, USA.

\author{
Shanshan Chen ${ }^{1,2}$, Amelia-Elena Rotaru ${ }^{1 *}$, Pravin Malla Shrestha ${ }^{1} \dagger$, Nikhil S. Malvankar $^{1,3}$, Fanghua Liu ${ }^{1,4}$, \\ Wei Fan ${ }^{5}$, Kelly P. Nevin' \& Derek R. Lovley'
}

'Department of Microbiology, University of Massachusetts, Amherst, MA 01003, USA, ${ }^{2}$ School of Environmental Science and Engineering, Sun Yat-sen University, Guangzhou 510275, China, ${ }^{3}$ Department of Physics, University of Massachusetts, Amherst, MA 01003, USA, ${ }^{4}$ Yantai Institute of Coastal Zone Research, Chinese Academy of Sciences, Yantai 264003, China, ${ }^{5}$ Department of Chemical Engineering, University of Massachusetts, Amherst, MA 01003, USA.

Biochar, a charcoal-like product of the incomplete combustion of organic materials, is an increasingly popular soil amendment designed to improve soil fertility. We investigated the possibility that biochar could promote direct interspecies electron transfer (DIET) in a manner similar to that previously reported for granular activated carbon (GAC). Although the biochars investigated were 1000 times less conductive than GAC, they stimulated DIET in co-cultures of Geobacter metallireducens with Geobacter sulfurreducens or Methanosarcina barkeri in which ethanol was the electron donor. Cells were attached to the biochar, yet not in close contact, suggesting that electrons were likely conducted through the biochar, rather than biological electrical connections. The finding that biochar can stimulate DIET may be an important consideration when amending soils with biochar and can help explain why biochar may enhance methane production from organic wastes under anaerobic conditions.

iochar, which is produced by heating biomass in a closed system with limited oxygen supply, has been promoted as an amendment to improve soil quality ${ }^{1,2}$, but the impact of biochar on soil microbial communities is poorly understood. Biochar has been used to enhance soil fertility ${ }^{3}$, remediate pollution, decrease green house gas emissions ${ }^{4}$ and sequester carbon in a worldwide quest to achieve carbon neutrality ${ }^{4}$. Biochar is hypothesized to increase the microbial soil content by concentrating organic substrates and nutrients on its surface ${ }^{5}$. Another feature of biochar that could potentially influence the activity of soil microbiota is that biochar is electrically conductive ${ }^{6}$. Conductive minerals/materials such as magnetite or GAC facilitated DIET by accelerating rates of anaerobic metabolism ${ }^{7-10}$. Under certain conditions, DIET is considered to be potentially more effective for interspecies electron transfer than strategies such as interspecies $\mathrm{H}_{2}$ or formate transfer that rely on the diffusion of electron carriers between species $^{11,12}$.

DIET has been shown possible in the absence of conductive materials via biological electrical connections such as pili ${ }^{13-17}$. For example, Geobacter metallireducens and Geobacter sulfurreducens grew in co-culture via DIET in a medium with ethanol as the electron donor and fumarate as the electron acceptor ${ }^{13}$. The two organisms needed to cooperate in order to metabolize ethanol and grow, because G. metallireducens can utilize ethanol as an electron donor, but is unable to use fumarate as an electron acceptor ${ }^{18}$, whereas G. sulfurreducens cannot use ethanol as an electron donor, but can reduce the electron acceptor, fumarate ${ }^{19}$. In co-culture, G. metallireducens produced acetate from ethanol and G. sulfurreducens utilized the electrons released from the ethanol oxidation as well as the acetate produced for fumarate reduction ${ }^{13}$. Multiple lines of evidence ${ }^{13-17}$ demonstrated that interspecies electron transfer in Geobacter co-cultures proceeded via DIET rather than $\mathrm{H}_{2}$ or formate transfer, and that the Geobacter pili, which posses metallic-like conductivity ${ }^{20,21}$, were required during DIET.

However, the initial adaption of Geobacter co-cultures to interspecies electron transfer via pili-mediated DIET took ca. 30 days $^{13}$. GAC substantially reduced the adaptation period for growth via DIET, with ethanol metabolism evident within a day ${ }^{9}$. Furthermore, the mechanism for DIET in the presence of GAC appeared to be different because strains of $G$. sulfurreducens deficient in pili or the pili-associated cytochrome OmcS, were incapable of DIET in the absence of $\mathrm{GAC}^{13}$, but functioned as well as wild-type in the presence of GAC ${ }^{9}$. Instead of forming close cell-to-cell connections as observed in the absence of $\mathrm{GAC}^{13}$, both species attached to the electrically conductive GAC suggesting that GAC served as a cell-to-cell conduit for electron flow between electron-donating and electron-accepting cells. GAC also accelerated electron transfer between G. metallireducens and M. barkeri promoting methane production from ethanol, and enhanced methane production was observed if aggregates from an anaerobic wastewater digester were amended with GAC ${ }^{9}$. 
Biochar, is a GAC precursor ${ }^{22}$ and chemical or physical activation of biochar yields GAC, with a higher surface area, porosity and increased aromaticity ${ }^{23,24}$. Electron conduction through a solid conductive medium is substantially different than molecular diffusion of shuttles. Conduction through solid materials involves migration of electrons in response to a difference in voltage potential whereas concentration gradients drive diffusion of shuttles. The term electron shuttle typically refers to soluble molecules such as humic substances $^{25}$, cysteine ${ }^{26}$, redox active metals ${ }^{27}$, or flavins ${ }^{28}$ that can accept electrons from one microorganism and donate them to another electron acceptor ${ }^{29}$ as a result of concentration gradients.

In this study we addressed the impact of biochar on syntrophic associations based on DIET. Syntrophic associations are at the root of carbon cycle in the environment, and this report unravels biochar as a potential contributor to methane emissions in the environment. We report on studies on the impact of biochar in defined co-cultures with $G$. metallireducens, an effective microorganism for evaluating DIET because it can donate electrons via DIET, and not by interspecies $\mathrm{H}_{2}$ or formate transfer ${ }^{13,14,16,18}$.

\section{Results}

Biochar stimulates DIET in Geobacter co-cultures. The addition of biochar to co-cultures of G. metallireducens and G. sulfurreducens in medium with ethanol as the electron donor and fumarate as the electron acceptor stimulated syntrophic metabolism of ethanol (Figure 1A) with the reduction of fumarate to succinate (Figure 1B) within two days. In contrast, a control co-culture of $G$. metallireducens and G. sulfurreducens, not amended with biochar, required ca. 30 days to adapt to ethanol metabolism ${ }^{13}$. Rates of ethanol loss and succinate production with a water-soluble extract of the biochar were only slightly faster than the biochar-free control (Figure 1), suggesting that the particulate fraction of the biochar was primarily responsible for stimulating co-culture metabolism.

The rates of ethanol metabolism varied somewhat with the biochar types, but this could not be correlated with the small differences in conductivity because the co-cultures amended with ESI biochar, which had the lowest conductivity (Table 1), metabolized ethanol at rates intermediate between the BEC and Kiln biochars, which both had conductivities ca. 2 -fold higher. The fast rates of ethanol metabolism in the presence of biochar were comparable with those previously observed with the same quantity of GAC ${ }^{9}$. However, the conductivity of GAC $(3000 \pm 327 \mu \mathrm{S} / \mathrm{cm})$ measured with the same method $^{9}$ was substantially higher than that of biochar (Table 1). On the other hand, a non-conductive material, glass beads, did not promote DIET under similar conditions ${ }^{9}$. In co-cultures amended with biochar acetate did not accumulate, and thus the oxidation of each mole of ethanol to carbon dioxide was coupled to the reduction of fumarate, the electron acceptor for G. sulfurreducens, and was expected to result in the production of six moles of succinate (reaction 1)

$\mathrm{C}_{2} \mathrm{H}_{6} \mathrm{O}+6 \mathrm{C}_{4} \mathrm{H}_{4} \mathrm{O}_{4}+3 \mathrm{H}_{2} \mathrm{O} \rightarrow 2 \mathrm{CO}_{2}+6 \mathrm{C}_{4} \mathrm{H}_{6} \mathrm{O}_{4}$ (reaction 1 )

The amounts of ethanol consumed and succinate produced in the 10 days of incubation (mean \pm standard deviation; $n=3$ ) in the presence of the BEC, ESI and Kiln biochars were $8.10 \pm 0.72 / 34.98 \pm$ $0.48 ; 6.98 \pm 0.47 / 33.37 \pm 0.24$; and $5.15 \pm 0.23 / 30.81 \pm 1.28$, respectively. Thus, the reduction of fumarate to succinate accounted for $72-88 \%$ of the electrons, which were derived from the ethanol removal in the biochar-amended cultures. In co-cultures amended with biochar, most $(78 \%)$ of the co-culture protein was firmly attached to the solid particles of biochar, after 10 days of incubation (Figure 2A). Quantitative PCR analysis of the attached cells indicated that the majority (69\%) were G. sulfurreducens (Figure 2B). Scanning electron microscopy revealed that attached cells did not form aggregates (Figure 3) like co-cultures without biochar, suggesting that direct biological connections between the cells were unnecessary.
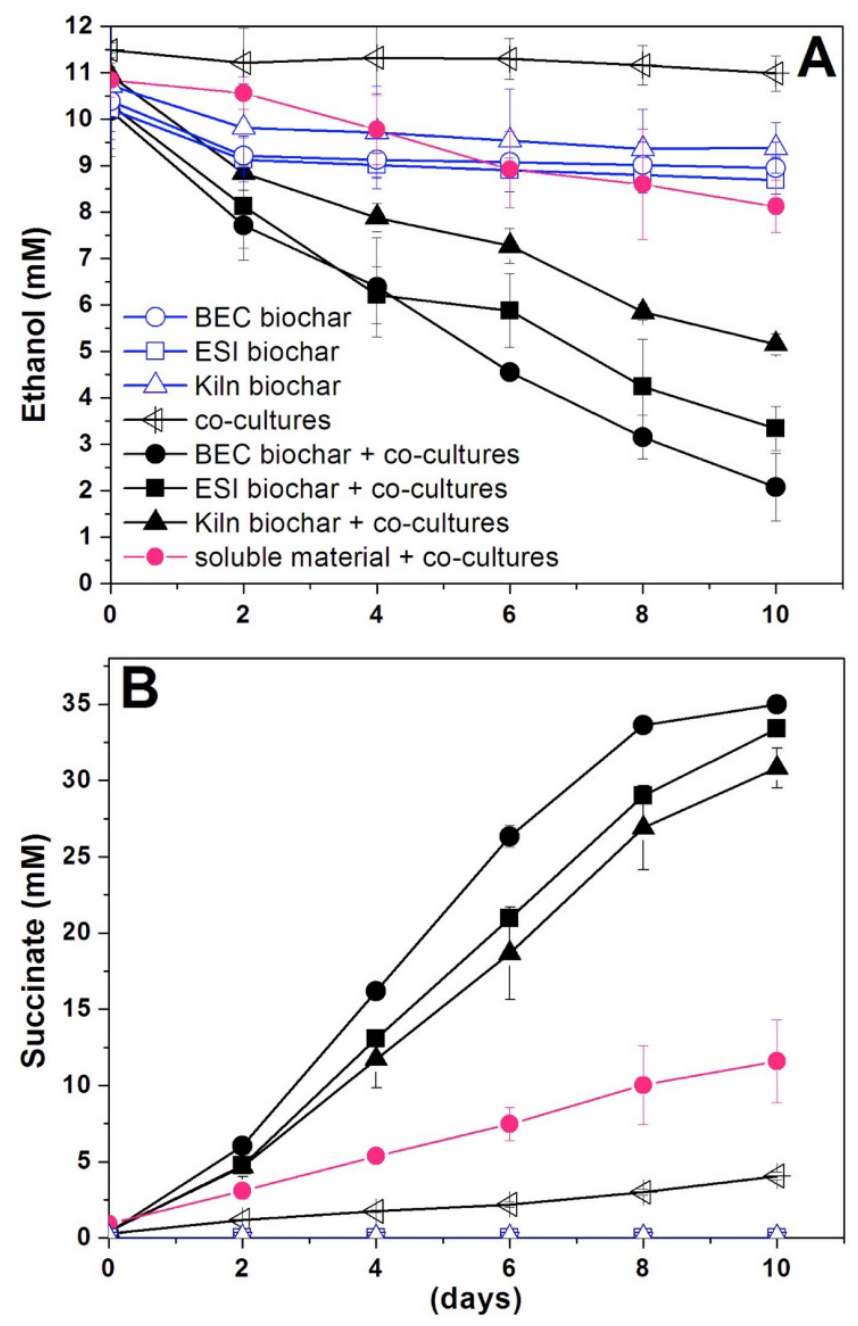

Figure 1 (A) Ethanol consumption and (B) succinate production with different types of biochar by a syntrophic co-culture of G. metallireducens and G. sulfurreducens. As control experiments we tested all different biochars with no cells, the co-culture without biochar and the co-culture with soluble components released from the biochar. The error bars represent standard deviations of the mean for triplicate cultures.

Cultures comprised solely of G. metallireducens slowly metabolized ethanol with a corresponding increase in acetate (Figure 4A), suggesting that biochar served as an electron acceptor (reaction 3 ).

$\mathrm{C}_{2} \mathrm{H}_{6} \mathrm{O}+\mathrm{H}_{2} \mathrm{O}+\left(\right.$ Biochar $\left._{\text {ox. }}\right) \rightarrow \mathrm{C}_{2} \mathrm{H}_{4} \mathrm{O}_{2}+4 \mathrm{H}^{+}+4 \mathrm{e}^{-}$ (Biochar ${ }_{\text {red. }}$ ) (reaction 2)

There was no ethanol metabolism in the absence of biochar (Figure 4B).

From the amount of ethanol metabolized over 10 days $(2.8 \mathrm{mmol} /$ $\mathrm{L} \times 0.01 \mathrm{~L}=0.028 \mathrm{mmol})$, and the stoichiometry of electron release from ethanol metabolism to acetate $(4 \mathrm{mmol}$ electrons $/ \mathrm{mmol}$ ethanol) it can be estimated that the $0.25 \mathrm{~g}$ of biochar in the culture tubes accepted 0.112 mmoles of electrons from G. metallireducens, which is ca. 0.5 mmoles of electrons accepted per gram of biochar. This is comparable to 0.35 mmoles of electrons that a gram of soil humic substances is capable of accepting during microbial reduction processes $^{30}$. However, unlike humic substances that, once reduced, can donate electrons to $\mathrm{Fe}(\mathrm{III})^{25,31}$, no $\mathrm{Fe}$ (III) was reduced when the reduced biochar was exposed to $\mathrm{Fe}(\mathrm{III})$ citrate, suggesting that, as previously demonstrated for $\mathrm{GAC}^{9}$, electron transport through biochar is unlikely to be attributed to quinone moieties, but rather to the conductive properties of the materials. Our Fe(III) citrate experiments with bioreduced biochar were carried out under physiological 
Table 1 | Physical and chemical properties of biochars

\begin{tabular}{|c|c|c|c|c|c|}
\hline Biochar & $\begin{array}{l}\text { Original } \\
\text { feedstock }\end{array}$ & Pyrolysis temperature $\left({ }^{\circ} \mathrm{C}\right)$ & $\begin{array}{l}\text { Particle size } \\
(\mathrm{mm})^{a}\end{array}$ & $\begin{array}{l}\text { BET surface } \\
\text { area }\left(\mathrm{m}^{2} / \mathrm{g}\right)^{b}\end{array}$ & $\begin{array}{l}\text { Electrical conductivity per g } \\
\text { biochar }(\mu \mathrm{S} / \mathrm{cm})^{c}\end{array}$ \\
\hline BEC & pine & 700 for $30 \mathrm{sec} \& 500$ for $15 \mathrm{~min}$ & $\leq 0.4$ & 15 & 4.41 \\
\hline ESI & pine & 500 for $2 \mathrm{~h}$ & $\leq 1$ & 167 & 2.11 \\
\hline
\end{tabular}

aMaximum particle size was estimated by inspection with transmission electron microscopy.

'The Brunauer-Emmett-Teller (BET) surface areas were obtained from $\mathrm{N}_{2}$ adsorption at $77 \mathrm{~K}$ with a Quantachrome Autosorb- ${ }^{41}$

The electrical conductivities were determined with a two-electrode system as previously described ${ }^{20}$.

conditions ( $\mathrm{pH}$ 6.5-7.0) unlike a previous study which reported that reduced activated carbon ${ }^{37}$ could reduce $\mathrm{Fe}(\mathrm{III})$ citrate at low $\mathrm{pH}$ conditions ${ }^{37}$.

Biochar stimulation of DIET in methanogenic co-cultures. Some methanogenic communities may exchange electrons via DIET ${ }^{16,32}$. DIET was recently confirmed in co-culture studies with G. metallireducens and Methanosaeta harundinacea ${ }^{16}$, a study which also demonstrated that G. metallireducens was unable to produce $\mathrm{H}_{2}$ or formate to provide electrons for $\mathrm{CO}_{2}$ reduction to methane by $\mathrm{H}_{2}$ / formate utilizing methanogenic partners like Methanospirillum hungatei or Methanobacterium formicicum ${ }^{16}$. The impact of conductive materials on DIET and methanogenesis in defined systems was apparent from studies with co-cultures of $G$. metallireducens and $M$. barkeri amended with $\mathrm{GAC}^{9}$. Amending co-cultures of G. metallireducens and M. barkeri with biochar stimulated the conversion of ethanol to methane (Figure 5A). There was a transient accumulation of acetate, but methane production was dependent on ethanol metabolism following reaction 2 .

$2 \mathrm{CH}_{3} \mathrm{CH}_{2} \mathrm{OH} \rightarrow 3 \mathrm{CH}_{4}+\mathrm{CO}_{2}$ (reaction 3)

In the presence of biochar the metabolism of $21 \pm 1 \mathrm{mM}$ ethanol (i.e. $210 \mu \mathrm{mol}$ in $10 \mathrm{ml}$ media) yielded $16 \pm 2 \mathrm{mmol} / \mathrm{l}$ methane (i.e. $272 \mu \mathrm{mol}$ in $17 \mathrm{ml}$ headspace). Thus, $86 \%$ of the electrons from ethanol metabolism were recovered in methane. In contrast, there was no ethanol metabolism or methane production in the G. metallireducens- $M$. barkeri co-cultures in the absence of biochar (Figure 5B) or with $M$. barkeri alone in the presence of biochar (Figure 5C). The water-soluble biochar fraction had only a minor stimulatory impact on ethanol metabolism in the G. metallireducens$M$. barkeri co-cultures, suggesting that the solid phase was the primary stimulatory component (Figure 5D).

Similar to Geobacter co-cultures, most of the cell protein $(71 \%)$ was firmly associated to the biochar particles in the methanogenic co-culture (Figure $6 \mathrm{~A}$ ) and $87 \%$ of the attached cells were G. metallireducens (Figure 6B). The higher abundance of $G$. metallireducens in the co-culture with M. barkeri, compared with the co-culture with $G$. sulfurreducens may be due to larger bio-volume of $M$. barkeri versus G. sulfurreducens, as apparent from SEM micrographs (Figure 3,7 ), as well as the lower energy yield from methane production versus fumarate reduction. Scanning electron microscopy revealed that $G$. metallireducens and M. barkeri attached to the biochar, but did not form aggregates with each other, suggesting that the electrical connections between the two species were through the biochar rather than via cell-to-cell electron transfer (Figure 7).

\section{Discussion}

The results demonstrate that biochar has sufficient conductivity to promote direct electron transfer between syntrophic partners in cocultures based on DIET. This provides a potential explanation for observations that some biochar amendments can enhance methane production in soils ${ }^{33}$ or in small-scale digesters converting organic waste to methane $\mathrm{e}^{34,35}$.

The results suggest that biochar promotes interspecies electron exchange via a conduction-based mechanism, in which electrons migrate through the biochar from electron-donating to electronaccepting cells. This is similar to the mechanism proposed for interspecies electron transfer through $\mathrm{GAC}^{9}$, but differs significantly from extracellular electron exchange with electron shuttles such as humic substances ${ }^{25}$. In the absence of conductive materials, microorganisms growing together, required a long adaption time and numerous transfers ${ }^{13}$, to get to the same substrate consumption rates as those observed with biochar or $\mathrm{GAC}^{9}$. This suggests that cells required time to express cellular components required for extracellular electron transfer ${ }^{14}$.

The ability of biochar to promote DIET with similar rates and stoichiometries as those observed in co-cultures amended with

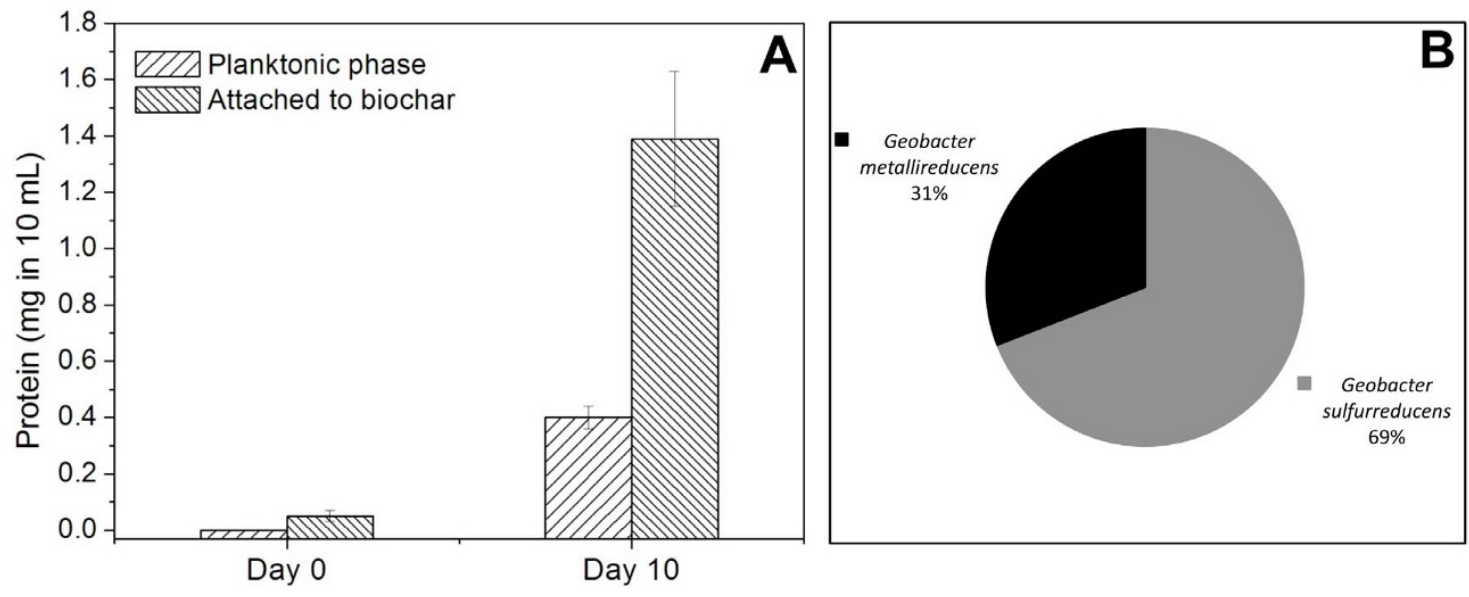

Figure 2 (A) Protein in $10 \mathrm{~mL}$ of medium on day 0 and day 10 in the planktonic phase and attached to BEC biochar, and (B) quantitative PCR analysis of the cells attached to the BEC biochar on day 10 in the G. metallireducens/G. sulfurreducens co-cultures. The error bars represent standard deviations of the mean for triplicate cultures. 


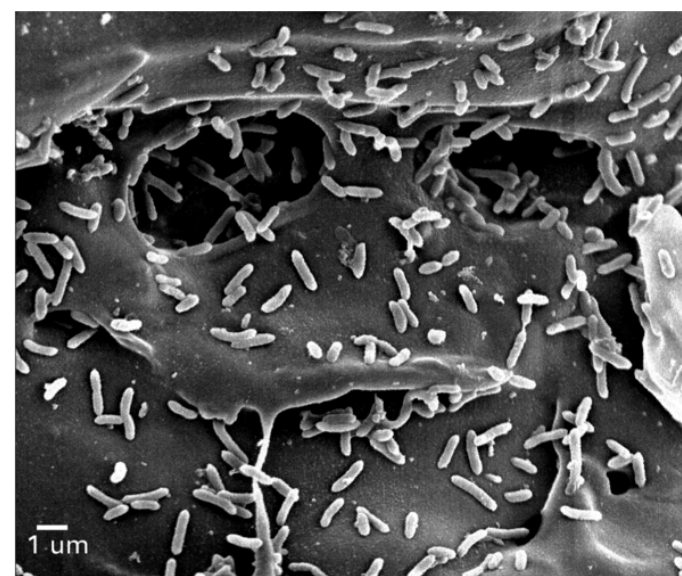

Figure 3 Scanning electron micrograph of one of the biochar tested (BEC) with a syntrophic co-culture of $G$. metallireducens and $G$. sulfurreducens.

$\mathrm{GAC}^{9}$, might be surprising considering the conductivity of biochar is 1000 -fold less than that of GAC. The higher conductivity of activated carbon is likely due to increased surface area and porosity and increased aromatization, which happens during the conversion of biochars into activated carbon at higher temperatures ${ }^{22,23}$. Aromaticity, a consequence of electron delocalization between aromatic rings localized on distinct neighboring planes, gives conductive properties to graphite, charcoals or other organic polymers ${ }^{24}$, and as discovered recently even to the pili of Geobacter species ${ }^{21}$. The conductivity of the biochars evaluated here was comparable to that of $G$. sulfurreducens pili preparations ${ }^{20,21}$, which are sufficient to effectively promote DIET. The ability of biochar to stimulate DIET appears to overcome the adaption period that cells require to begin expressing high levels of the components that are required for pili-based DIET $^{13,14,16}$. Materials with increased aromaticity are doped by reduction or oxidation reactions ${ }^{36}$. If the acceptor microorganism reduces sections of the biochar and the donor microorganism oxidizes sections of the biochar, there will be intrinsic charge differences between sections of biochar, promoting electron flow. This has been also noted on activated carbon, which accepted electrons from microorganisms and then released the electrons to $\mathrm{Fe}$ (III) citrate under acidic conditions $^{38}$.

However, biochar is a complex material and can modify environments to which it is added with properties other than conductivity. For example, biochar was speculated to act as a "shuttle" to mitigate $\mathrm{N}_{2} \mathrm{O}$ emissions during denitrification in soils ${ }^{38}$. Whereas other studies suggested that $\mathrm{N}_{2} \mathrm{O}$ formation in soils is due to abiotic processes happening on biochars surface enriched in surface charged groups, like quinones, metal ions or radicals ${ }^{39,40}$.

Our observations that biochar increases methane production in defined co-culture systems, in which partners were capable of direct electron transfer, changes the present understanding that biochar could mitigate methane gas emissions. Considering the potential significant impact of methane production on global warming, and the persistence of biochar in soil, warrants further long-term studies on how soil methanogenic communities are affected by biochar amendments and the impact of biochar on the global carbon cycle.

\section{Methods}

Characterization of biochars. Three pine biochars that differed somewhat in their mode of production, particle size, and surface area were evaluated (Table 1). All biochars were sieved (pore size $3 \mathrm{~mm}$ ), and the size of particles was inspected with a transmission electron microscope. The Brunauer-Emmett-Teller (BET) surface areas of the other three kinds of biochar were calculated from the $\mathrm{N}_{2}$ adsorption and desorption isotherms at $77^{\circ} \mathrm{K}$ obtained using Quantachrome Autosorb-1 as previously described ${ }^{41}$. The electrical conductivities of biochar were determined by two-probe electrical conductance measurements using two gold electrodes separated
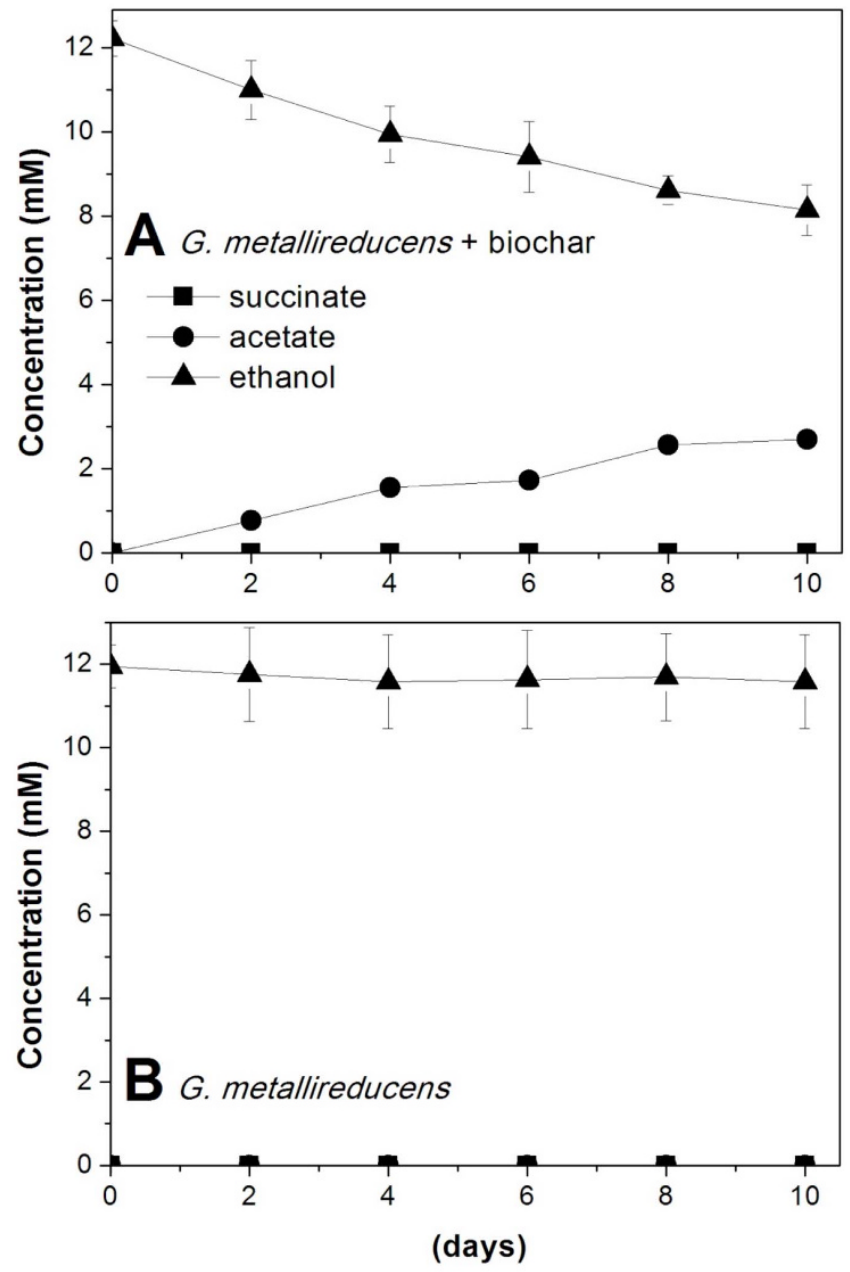

Figure $4 \mid$ Ethanol consumption, succinate and acetate production in medium with ethanol as the electron donor with (A) G. metallireducens and the BEC biochar or (B) G. metallireducens alone. The error bars represent standard deviations of the mean for triplicate cultures.

by $50 \mu \mathrm{m}$ non-conductive gap, as previously described ${ }^{13,20}$. Biochar was placed between the two gold electrodes to bridge the non-conductive gap. Voltage was applied using a Keithley 2400 sourcemeter. Voltage was scanned from $0 \mathrm{~V}$ to $+0.05 \mathrm{~V}$ in steps of $0.025 \mathrm{~V}$. For each sample, current was measured 100 seconds after setting the voltage to allow the exponential decay of the transient ionic current in the gap and to measure steady state electronic current ${ }^{42}$. We collected data with the Labview data acquisition system (National Instruments, TX, USA), and data analyses were performed with Igor Pro (Wavemetrics Inc., OR USA).

Microorganisms, media and growth conditions. Geobacter metallireducens strain GS-15 (ATCC 53774), Geobacter sulfurreducens strain PCA (ATCC 51573), and Methanosarcina barkeri type strain, DSM 800 (ATCC 43569) were obtained from our laboratory culture collection. Prior to initiating the co-cultures, G. metallireducens was maintained in a medium with ethanol $(10 \mathrm{mM})$ as the electron donor and $\mathrm{Fe}$ (III) citrate $(55 \mathrm{mM})$ as the electron acceptor as previously described ${ }^{17}$. G. sulfurreducens was cultured routinely with $10 \mathrm{mM}$ acetate as electron donor and $40 \mathrm{mM}$ fumarate as electron acceptor in a fresh water mineral medium as previously described ${ }^{17} . M$. barkeri was maintained on DSMZ methanogenic medium 120 with $30 \mathrm{mM}$ acetate as methanogenic substrate ${ }^{9}$. All pure cultures and co-cultures were incubated anaerobically, in $27 \mathrm{~mL}$ pressure tubes with $10 \mathrm{~mL}$ medium under an anoxic atmosphere of $80: 20$ of $\mathrm{N}_{2}: \mathrm{CO}_{2}$ as previously described ${ }^{13,17}$.

Co-cultures of $G$. metallireducens and G. sulfurreducens were initiated as previously described ${ }^{9,17}$ by introducing a $5 \%$ inocula of each microorganism into a medium with ethanol $(10 \mathrm{mM})$ as the electron donor and fumarate $(40 \mathrm{mM})$ as the electron acceptor. The incubation temperature was $30^{\circ} \mathrm{C}$. Co-cultures of G. metallireducens and $M$. barkeri were initiated with a $5 \%$ inocula of each microorganism into DSMZ methanogenic medium 120 with $20 \mathrm{mM}$ ethanol as the electron donor ${ }^{9}$. Incubations of the methanogenic cocultures were done at $37^{\circ} \mathrm{C}$. All experiments were carried on with three biological replicates.

In order to test the effect of biochar amendments, $0.25 \mathrm{~g}$ of biochar was added to $0.5 \mathrm{ml}$ of the appropriate co-culture medium, under $\mathrm{N}_{2}: \mathrm{CO}_{2}$ and the medium was 

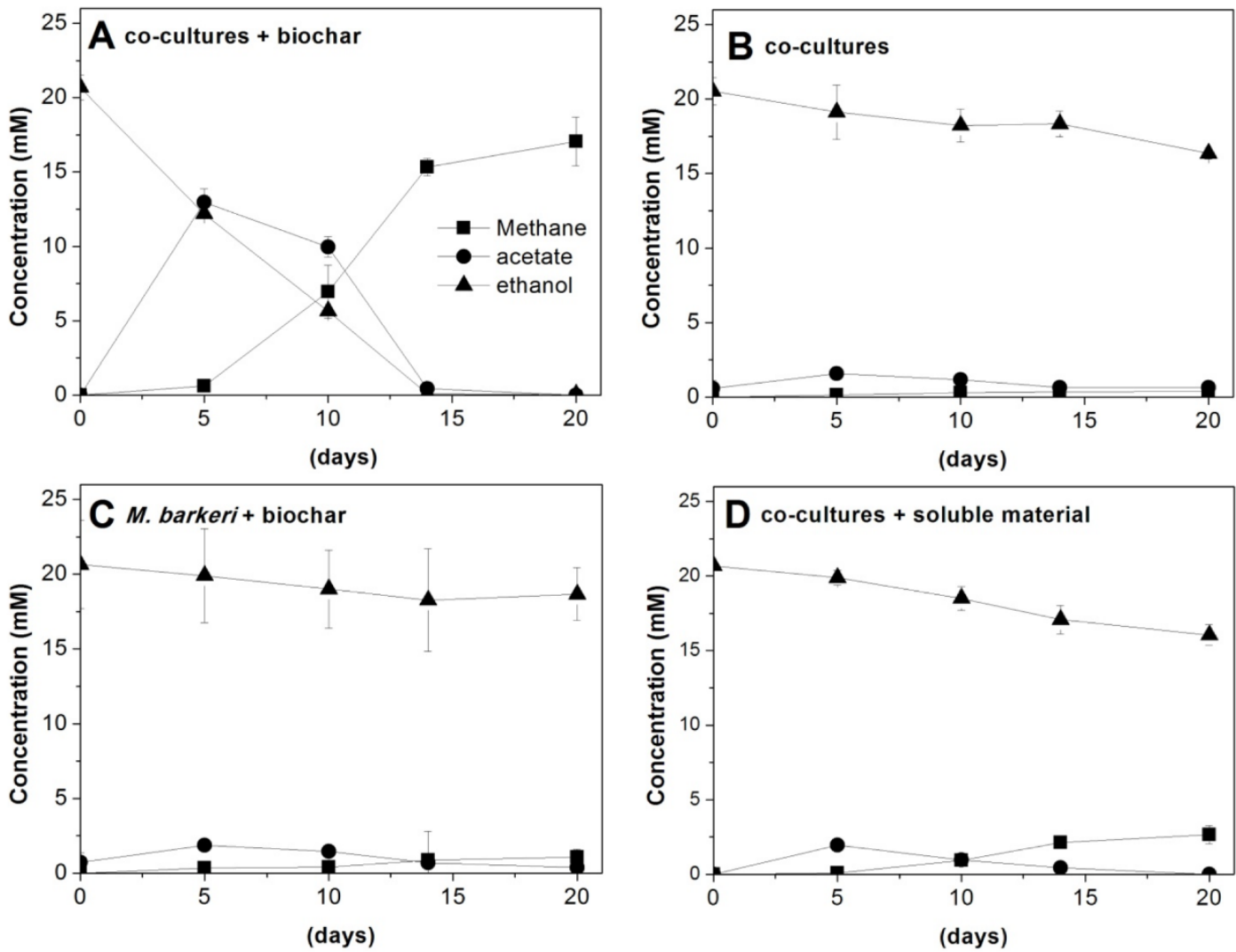

Figure 5 Ethanol consumption, methane and acetate production in (A) co-cultures of G. metallireducens-M. barkeri with the BEC biochar, (B) unamended co-cultures of G. metallireducens-M. barkeri (C) and pure culture of M. barkeri with the BEC biochar, and (D) co-cultures of G. metallireducens-M. barkeri with the soluble components released from the BEC biochar. The error bars represent standard deviations of the mean for triplicate cultures.

autoclaved for 30 minutes. Additional sterile medium $(9 \mathrm{ml})$ was added under anaerobic conditions, while ethanol and cells were inoculated afterwards.

Water extracts of biochar were obtained by incubating $0.25 \mathrm{~g}$ of biochar in $9 \mathrm{ml}$ of culture medium on a shaker under sterile, anaerobic conditions for either 10 days $(G$. metallireducens - G. sulfurreducens co-cultures) or 20 days (G. metallireducens - $M$. barkeri co-cultures) to replicate the length of time that the co-cultures were exposed to biochar. Ethanol and cells were added afterwards.

Quantitative PCR analysis. DNA was extracted from the cells attached to biochar from triplicate $10 \mathrm{ml}$ co-cultures as previously described ${ }^{9}$. To determine the specific abundance of cells in co-cultures amended with biochar, quantitative PCR was performed using G. metallireducens specific primers (Gmet_F 5'-TGGCCCACATCTTCATCTC-3', Gmet_R 5'-TGCATGTTTTCATCCACGAT-3'), versus primers universal to both G. sulfurreducens and G. metallireducens (Geo16S_F 5'-GAGGTACCGTCAAGACCAA-3', Geo16S_R 5'-GCCACACTG-

GAACTGAGACA-3'), or specific for Methanosarcina species (MB16S_F 5' GGGTCTAAAGGGTCCGTAGC-3', MB16S_R 5' GTTCTGGTAAGACGCCT-

TCG-3'), depending on the type of co-culture tested. Before conducting the quantitative PCR experiment primer pairs were validated for the primer efficiency. The quantitative PCR was carried out using Fast Syber green master mix (ABI) in Real time PCR cycler (ABI-9500) following the manufacturer's protocol.

Scanning electron microscopy. In order to evaluate cell attachment to biochar, the biochar-attached fraction was studied by scanning electron microscopy at the end of the co-culture growth (day 10 of the G. metallireducens-G.sulfurreducens cycle, and day 20 of the G. metallireducens-M. barkeri). Samples were first fixed with $2.5 \%$
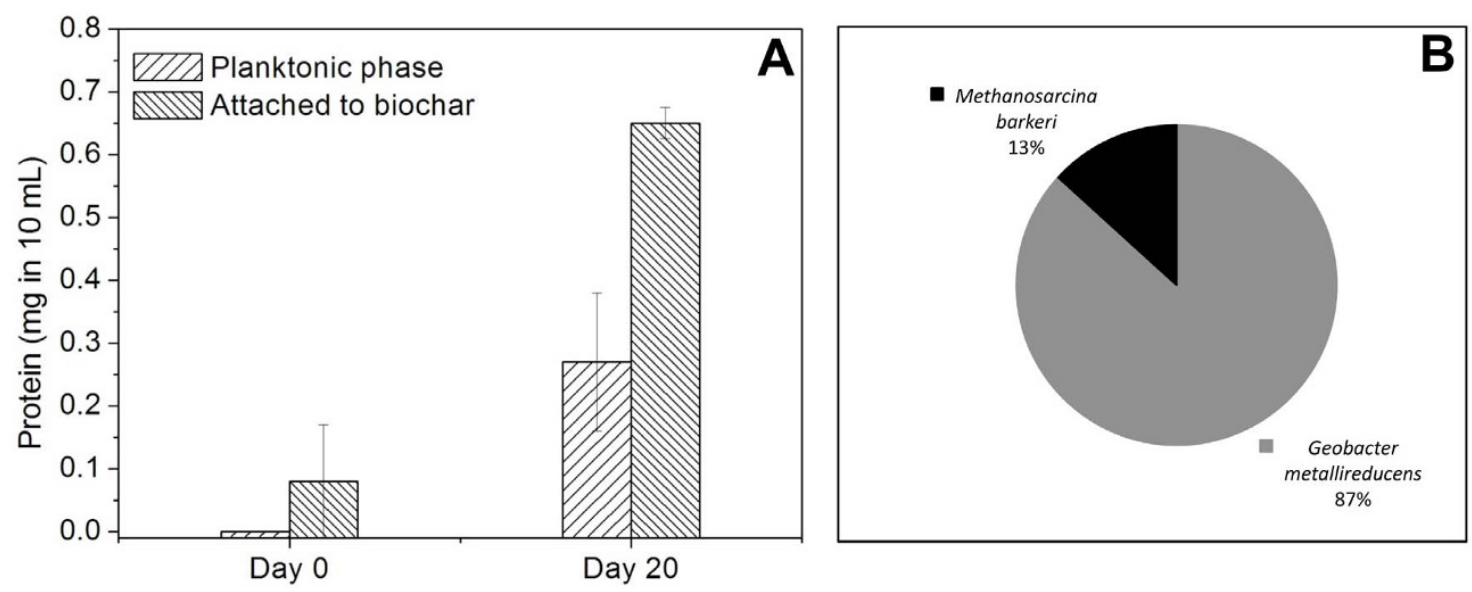

Figure 6 (A) Protein in $10 \mathrm{~mL}$ of medium on day 0 and day 20 in the planktonic phase and attached to BEC biochar, and (B) Quantitative PCR analysis of the cells attached to the BEC biochar on day 20 in the G. metallireducens/M. barkeri co-cultures. The error bars represent standard deviations of the mean for triplicate cultures. 


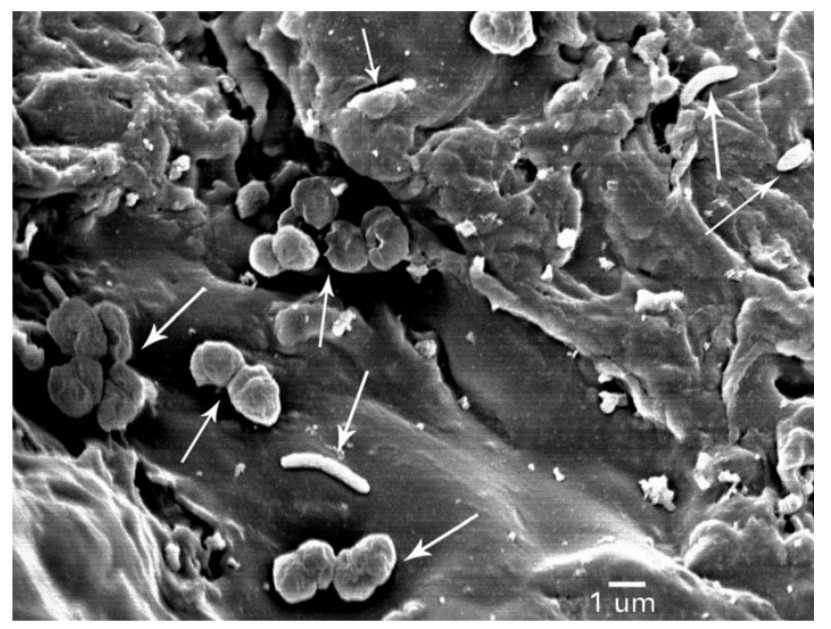

Figure 7 | Scanning electron micrograph of a syntrophic co-culture of $G$. metallireducens (rods) and M. barkeri (spheres) with the BEC biochar. The white arrow points to the representative cells.

glutaraldehyde in $0.1 \mathrm{M}$ phosphate buffer for up to 12 hours at $4^{\circ} \mathrm{C}$, then washed 3 times in $0.1 \mathrm{M}$ phosphate buffer at $4^{\circ} \mathrm{C}$ for $10 \mathrm{~min}$ each, dehydrated further in an ethanol/water mixture of $50 \%, 70 \%, 80 \%, 90 \%, 95 \%$ and $100 \%$ for 10 minutes each (dehydration in 100\% ethanol was done 3 times), and at last immersed twice for 30 seconds in pure hexamethyldisilazane (Sigma Aldrich, St Louis, MO, USA) followed by 10 minutes of air-drying ${ }^{43}$.

Analytical techniques. Subsamples for gas chromatographic analysis of methane and ethanol and high performance liquid chromatography analysis of acetate, fumarate, formate and succinate were withdrawn and processed as previously described ${ }^{32}$. In order to further support biomass attachment onto biochar at the end of co-culture incubations, the liquid fraction was separated from the biochar by lysis of the biochar in $0.5 \mathrm{~N} \mathrm{NaOH}$ and bead beating for 30 seconds with sterile glass beads to ensure cell disruption. Cell protein in the culture supernatant and biochar fractions was determined using the bicinchoninic acid method ${ }^{44}$ with bovine serum albumin (BSA) as protein standard.

1. Lehmann, J. A handful of carbon. Nature 447, 143-144 (2007).

2. Zheng, H. et al. Characteristics and nutrient values of biochars produced from giant reed at different temperatures. Bioresour. Technol. 130, 463-471 (2013).

3. Chan, K., Van Zwieten, L., Meszaros, I., Downie, A. \& Joseph, S. Using poultry litter biochars as soil ammendments. Soil Res. 46, 437-444 (2008).

4. Woolf, D., Amonette, J. E., Street-Perrott, F. A., Lehmann, J. \& Joseph, S Sustainable biochar to mitigate global climate change. Nat. Commun. 1, 56 (2010).

5. Lehmann, J. et al. Biochar effects on soil biota: A review. Soil Biol. Biochem. 43, 1812-1836 (2011).

6. Xu, W., Pignatello, J. \& Mitch, W. Role of black carbon electrical conductivity in mediating hexahydro-1,3,5-triazine (RDX) transformation on carbon surfaces by sulfides. Environ. Sci. Technol. 34, 2472-2478 (2013).

7. Kato, S., Hashimoto, K. \& Watanabe, K. Methanogenesis facilitated by electric syntrophy via (semi)conductive iron-oxide minerals. Environ. Microbiol. 14, 1646-1654 (2012).

8. Kato, S., Hashimoto, K. \& Watanabe, K. Microbial interspecies electron transfer via electric currents through conductive minerals. Proc. Natl. Acad. Sci. USA 109, 10042-10046 (2012).

9. Liu, F. et al. Promoting direct interspecies electron transfer with activated carbon. Energy Environ. Sci. 5, 8982-8989 (2012).

10. Liu, F. et al. Magnetite compensates for the lack of a pilin-associated c-type cytochrome in extracellular electron transfer. Environ. Microbiol. DOI: 10.1111/ 1462-2920.12485. (2014).

11. Lovley, D. R. Live wires: direct extracellular electron exchange for bioenergy and the bioremediation of energy-related contamination. Energy Environ. Sci. 4, 4896-4906 (2011).

12. Lovley, D. R. Electromicrobiology. Annu. Rev. Microbiol. 66, 391-409 (2012).

13. Summers, Z. M. et al. Direct exchange of electrons within aggregates of an evolved syntrophic coculture of anaerobic bacteria. Science 330, 1413-1415 (2010).

14. Shrestha, P. M. et al. Transcriptomic and genetic analysis of direct interspecies electron transfer. Appl. Environ. Microbiol. 79, 2397-2404 (2013).

15. Shrestha, P. M. et al. Syntrophic growth with direct electron transfer as the sole mechanism for for energy exchange. Environ. Microbiol. Rep. 5, 904-910 (2013).

16. Rotaru, A.-E. et al. A new model for electron flow during anaerobic digestion: direct interspecies electron transfer to Methanosaeta for the reduction of carbon dioxide to methane. Energy Environ. Sci. 7, 408-415 (2014).
17. Rotaru, A.-E. et al. Interspecies electron transfer via hydrogen and formate rather than direct electrical connections in co-cultures of Pelobacter carbinolicus and Geobacter sulfurreducens. Appl. Environ. Microbiol. 78, 7645-7651 (2012).

18. Lovley, D. R. et al. Geobacter metallireducens gen. nov. sp. nov., a microorganism capable of coupling the complete oxidation of organic compounds to the reduction of iron and other metals. Arch. Microbiol. 159, 336-344 (1993).

19. Caccavo, F., Jr. et al. Geobacter metallireducens sp. nov., a hydrogen- and acetateoxidizing dissimilatory metal-reducing microorganism. Appl. Environ. Microbiol. 60, 3752-3759 (1994)

20. Malvankar, N. S. et al. Tunable metallic-like conductivity in microbial nanowire networks. Nat. Nanotechnol. 6, 573-579 (2011).

21. Vargas, M. et al. Aromatic amino acids required for pili conductivity and longrange extracellular electron transport in Geobacter sulfurreducens. mBio 4, e00105-13 (2013).

22. Azargohar, R. \& Dalai, A. in 27th Symposium on Biotechnology for Fuels and Chemicals, Vol. 129-132. (eds. McMillan, J., Adney, W., Mielenz, J. \& Klasson, K.) 762-773 (Humana Press, Denver, CO, USA; 2006).

23. Lehmann, J. \& Joseph, S. (eds.) Biochar for environmental management: science and technology. (Earthscan, London, Sterling, VA; 2009).

24. Bourke, J. et al. Do all carbonized charcoals have the same chemical structure? 2. A model of the chemical structure of carbonized charcoal. Ind. Eng. Chem. Res. 46, 5954-5967 (2007).

25. Lovley, D. R., Coates, J. D., Blunt-Harris, E. L., Phillips, E. J. \& Woodward, J. C. Humic substances as electron acceptors for microbial respiration. Nature 382, $445-448$ (1996).

26. Kaden, J., Galushko, A. \& Schink, B. Cysteine-mediated electron transfer in syntrophic acetate oxidation by cocultures of Geobacter sulfurreducens and Wolinella succinogenes. Arch. Microbiol. 178, 53-58 (2002).

27. Nevin, K. P. \& Lovley, D. R. Potential for nonenzymatic reduction of Fe (III) via electron shuttling in subsurface sediments. Environ. Sci. Technol. 34, 2472-2478 (2000).

28. Hernandez, M. \& Newman, D. Extracellular electron transfer. Cell. Mol. Life Sci. 58, 1562-1571 (2001)

29. Lovley, D. R. Bug juice: harvesting electricity with microorganisms. Nat. Rev. Microbiol. 4, 497-508 (2006).

30. Lovley, D. et al. Humic substances as a mediator for microbially catalyzed metal reduction. Acta Hydrochim. Hydrobiol. 26, 152-157 (1998).

31. Scott, D. T., McKnight, D. M., Blunt-Harris, E. L., Kolesar, S. E. \& Lovley, D. R. Quinone moieties act as electron acceptors in the reduction of humic substances by humics-reducing microorganisms. Environ. Sci. Technol. 32, 2984-2989 (1998).

32. Morita, M. et al. Potential for direct interspecies electron transfer in methanogenic wastewater digester aggregates. mBio 2, e00159-00111 (2011).

33. Yu, L., Tang, J., Zhang, R., Wu, Q. \& Gong, M. Effects of biochar application on soil methane emission at different soil moisture levels. Biol. Fert. Soils 49, 119-128 (2013).

34. Inthapanya, S., Preston, T. \& Leng, R. Biochar increases biogas production in a batch digester charged with cattle manure. Livest. Res. Rural Dev. 24, Article \#212 (2012).

35. Das, K., Balagursamy, N., Chinnasamy, S., Martinez Castro, G. J. \& Espino Lopez, C. in $W O / 2011 / 019871$. (ed. WO Patent 2, 019,871) (WO Patent 2, 011,019,871, WO Patent 2,011,019,871; 2011).

36. Snook, G. A., Kao, P. \& Best, A. S. Conducting-polymer-based supercapacitor devices and electrodes. J. Power Sources 196, 1-12 (2011).

37. van der Zee, F. P., Bisschops, I. A. E., Lettinga, G. \& Field, J. A. Activated Carbon as an Electron Acceptor and Redox Mediator during the Anaerobic Biotransformation of Azo Dyes. Environ. Sci. Technol. 37, 402-408 (2002),

38. Cayuela, M. L. et al. Biochar and denitrification in soils: when, how much and why does biochar reduce $\mathrm{N} 2 \mathrm{O}$ emissions? Sci. Rep. 3 (2013).

39. Joseph, S. D. et al. An investigation into the reactions of biochar in soil. Soil Res. 48, 501-515 (2010).

40. Heymann, K., Lehmann, J., Solomon, D., Schmidt, M. W. I. \& Regier, T. C 1 s Kedge near edge X-ray absorption fine structure (NEXAFS) spectroscopy for characterizing functional group chemistry of black carbon. Org. Geochem. $\mathbf{4 2}$, 1055-1064 (2011).

41. Fan, W. et al. Hierarchical nanofabrication of microporous crystals with ordered mesoporosity. Nature Mater. 7, 984-991 (2008).

42. Du, K. et al. Self-Assembled Electrical Contact to Nanoparticles Using Metallic Droplets. Small 5, 1974-1977 (2009).

43. Araujo, J. C. et al. Comparison of hexamethyldisilazane and critical point drying treatments for SEM analysis of anaerobic biofilms and granular sludge. J. Electron. Microsc. 52, 429-433 (2003).

44. Smith, P. et al. Measurement of protein using bicinchoninic acid. Anal. Biochem. 150, 76-85 (1985).

\section{Acknowledgments}

We thank Trevor Woodard for facilitating HPLC and GC analysis; Louis Raboin for facilitating SEM analysis; Kenneth $\mathrm{H}$. Williams and Baoshan Xing for providing biochar. The first author thanks the Oversea Study Program of Guangzhou Elite Project and the Innovative Dotorial Candidates Training Project of Sun Yat-sen University. This research 
was supported by the Office of Science (BER), U. S. Department of Energy, Award No. DESC0004485

\section{Author contributions}

D.R.L., A.E.R. and S.C. conceived the experiments. S.C. performed the experiments with help from A.E.R., F.L. and K.P.N. P.M.S. performed qPCR experiments. N.S.M. performed the conductivity measurements. W.F. characterized the biochars. D.R.L., S.C. and A.E.R analyzed the data and wrote the manuscript. All authors have seen the manuscript at all stages, discussed the data and agreed to the content.

\section{Additional information}

Competing financial interests: The authors declare no competing financial interests. How to cite this article: Chen, S. et al. Promoting Interspecies Electron Transfer with Biochar. Sci. Rep. 4, 5019; DOI:10.1038/srep05019 (2014).

(c) (i) (-) This work is licensed under a Creative Commons Attribution-NonCommercialShareAlike 3.0 Unported License. The images in this article are included in the article's Creative Commons license, unless indicated otherwise in the image credit; if the image is not included under the Creative Commons license, users will need to obtain permission from the license holder in order to reproduce the image. To view a copy of this license, visit http://creativecommons.org/licenses/by-nc-sa/3.0/ 